\title{
Konditionierung mit Ganzkörperbestrahlung oder Chemotherapie bei ALL im Kindesalter
}

\author{
Eine multinationale, randomisierte Phase-III-Studie auf Nichtunterlegenheit
}

\author{
Martin G. Sauer ${ }^{1}$ \\ Angenommen: 8. Juni 2021 / Online publiziert: 2. Juli 2021 \\ (c) Der/die Autor(en) 2021
}

Fragestellung Die Ganzkörperbestrahlung (TBI) als Bestandteil der Konditionierung vor allogener Blutstammzelltransplantation (HSCT) ist effektiv bei Kindern mit Hochrisiko-ALL. Die Spätfolgen der Bestrahlung können allerdings substanziell sein. Deswegen wurde die Frage gestellt, ob eine reine Chemokonditionierung die Ganzkörperbestrahlung ersetzen kann.

Patienten und Methoden Die Studie (Akronym: FORUM) wurde als randomisierte, kontrollierte, internationale openlabel Phase-III-Studie auf Nichtunterlegenheit konzipiert und multizentrisch durchgeführt. Kinder und Jugendliche, die sich zum Zeitpunkt der HSCT in kompletter Remission befanden, 4-21 Jahre alt waren und einen HLA-kompatiblen Spender hatten, wurden zwischen einer Konditionierung mit 12 Gy TBI und Etoposid oder einer reinen Chemokonditionierung mit Fludarabin, Thiotepa und entweder Treosulfan oder Busulfan randomisiert. Die Schwelle für Nichtunterlegenheit wurde auf $8 \%$ festgesetzt. Bei 1000 zu randomisierenden Patienten innerhalb von 5 Jahren, einer Nachbeobachtung von mindestens 2 Jahren und einem alpha-Fehler von $5 \%$ wurde eine Aussagekraft von $80 \%$ errechnet. Ein frühzeitiger Studienabbruch sollte in dem Fall

Originalpublikation Peters C, Dalle JH, Locatelli F, Poetschger U et al (2021) Total body irradiation or chemotherapy conditioning in childhood ALL: a multinational, randomized, noninferiority phase III study. J Clin Oncol 39:295-307

Prof. Dr. med. Martin G. Sauer

sauer.martin@mh-hannover.de

1 Bereich Pädiatrische Stammzelltransplantation und Zelltherapie, Klinik für Pädiatrische Hämatologie und Onkologie, Medizinische Hochschule Hannover, Carl-Neuberg-Straße 1, OE 6780 Hannover, Deutschland erfolgen, wenn sich die reine Chemokonditionierung der TBI im Verlauf unterlegen zeigen sollte.

Ergebnisse Zwischen April 2013 und Dezember 2018 wurden 417 Patienten randomisiert, 212 in die TBI- und 201 in die Chemotherapiegruppe. Im März 2019 musste die Studie vorzeitig abgebrochen werden. Die mediane Nachbeobachtungszeit lag zu dieser Zeit bei 2,1 Jahren. Das Überleben nach zwei Jahren war in der TBI-Gruppe signifikant besser als in der Chemotherapiegruppe (91\% vs. $75 \% ; p<0,0001)$. Die kumulative Inzidenz für ein Rezidiv und die therapieassoziierte Letalität nach TBI lagen bei $12 \%$ bzw. $2 \%$ und nach Chemokonditionierung bei $33 \%$ bzw. $9 \%(p<0,0001$ bzw. $p=0,269)$.

Schlussfolgerung der Autoren Aufgrund des signifikant besseren Gesamtüberlebens und einer niedrigeren Rezidivrate nach einer Konditionierung mit TBI und Etoposid im Vergleich zur reinen Chemokonditionierung wird erstere zukünftig bei Kindern mit HR-ALL empfohlen, die älter als 4 Jahre sind.

\section{Kommentar}

Die Bestrahlung als hochwirksame Therapiekomponente in der Behandlung der akuten lymphatischen Leukämie konnte über die vergangenen Jahrzehnte zunehmend durch intensivierte Chemotherapieregimen verlassen werden. Dieses führte unter Erhalt guter rezidivfreier Überlebensraten zu einer signifikanten Reduktion strahlentherapieinduzierter Spätfolgen wie Wachstumsdefizienz, einer anhaltenden Reduktion der kognitiven Leistungsfähigkeit, Störungen der zentralen Hormonsteuerung, Ausbildung von Katarakten und Zweitmalignomen [1]. Busulfan mit seiner aus- 
gezeichneten Liquorgängigkeit und seinen myeloablativen Eigenschaften wurde in der Transplantation bereits früh als Ersatz für eine Ganzkörperbestrahlung (TBI) eingeführt und deshalb auch gelegentlich als „liquid radiation“ bezeichnet. In der klassischen Kombination mit Cyclophosphamid war Busulfan aber mit nicht unerheblichen Akuttoxizitäten verbunden. In der Tat schien eine große Metaanalyse eine signifikant höhere therapieassoziierte Letalität nach busulfanbasierten Konditionierungsregimen zu belegen [2]. Auch für die TBI ist bzw. war der klassische Kombinationspartner Cyclophospahmid. Eine ursprünglich in Deutschland pilotisierte und später in die USA (City of Hope) überführte Kombination einer TBI mit Etoposid war mit vergleichbarem leukämiefreien Überleben und relativ guter Verträglichkeit assoziiert und deshalb Jahre später konzeptionell für die Pädiatrie übernommen und im Rahmen des BerlinFrankfurt-Münster-Studienverbundes wieder nach Europa repatriiert worden.

Im ALL-SCT-BFM-2003-Trial wurde damit bei guter Leukämiekontrolle eine bis dahin unerreicht niedrige therapieassoziierte Letalität im einstelligen Prozentbereich erzielt [3]. Inzwischen waren Fludarabin und Thiotepa als relativ gut tolerierte und antileukämisch hochwirksame Agentien als Partner für Busulfan in reinen Chemokonditionierungen bei ALL verwendet worden. Treosulfan als dem Busulfan ähnliche, aber einfacher zu handhabende Substanz, verdrängte in einigen europäischen Ländern zunehmend das Busulfan in der HSCT [4]. Die sich nun aufdrängende wichtige Frage, ob diese wirksamen und relativ gut verträglichen Chemokonditionierungen in Zukunft eine TBI-haltige Konditionierung bei Kindern mit ALL ersetzen und damit entscheidend zur Verringerung strahlenassoziierter Nebenwirkungen nach HSCT beitragen könnten, muss nach dieser wichtigen randomisierten Studie wegen der signifikant schlechteren Leukämiekontrolle ohne TBI als negativ beantwortet werden.

\section{Fazit}

Dies ist die erste randomisiert durchgeführte Studie, die prospektiv eine TBI-basierte vs. eine reine Chemokonditionierung (beide myeloablativ) hinsichtlich eines leukämiefreien Überlebens nach HSCT vergleicht. Bei Kindern und Jugendlichen mit ALL erzielt eine Konditionierung mit Ganzkörperbestrahlung (12 Gy, aufgeteilt auf 6 Fraktionen über 3 Tage) in Kombination mit Etoposid $\left(1,8 \mathrm{~g} / \mathrm{m}^{2}\right.$; Höchstdosis: 3,6g) eine signifikant bessere Leukämiekontrolle bei tendenziell besserer Akutverträglichkeit als eine Chemokonditionierung, bestehend aus Fludarabin $\left(30 \mathrm{mg} / \mathrm{m}^{2}\right.$ einmal täglich an 5 aufeinanderfolgenden Ta- gen), Thiotepa ( $5 \mathrm{mg} / \mathrm{kg}$ zweimal am Tag an einem Tag) und entweder Treosulfan $\left(14 \mathrm{~g} / \mathrm{m}^{2}\right.$ einmal am Tag an 3 aufeinanderfolgenden Tagen) oder gewichtsadaptiertes Busulfan (an 4 aufeinanderfolgenden Tagen). Interessanterweise verlaufen dabei die Kurven für leukämiefreies Überleben mit dem gut liquorgängigen Busulfan im Vergleich mit dem wenig ZNS-gängigen Treosulfan fast identisch.

Diese Studie muss als Meilenstein in der hämatopoetischen Stammzelltransplantation angesehen werden und wird die Verwendung der Ganzkörperbestrahlung bei der pädiatrischen ALL auf Jahre hinaus als State of the Art festlegen.

\section{Martin G. Sauer, Hannover}

Funding Open Access funding enabled and organized by Projekt DEAL.

Interessenkonflikt M.G. Sauer gibt an, dass kein Interessenkonflikt besteht.

Open Access Dieser Artikel wird unter der Creative Commons Namensnennung 4.0 International Lizenz veröffentlicht, welche die Nutzung, Vervielfältigung, Bearbeitung, Verbreitung und Wiedergabe in jeglichem Medium und Format erlaubt, sofern Sie den/die ursprünglichen Autor(en) und die Quelle ordnungsgemäß nennen, einen Link zur Creative Commons Lizenz beifügen und angeben, ob Änderungen vorgenommen wurden.

Die in diesem Artikel enthaltenen Bilder und sonstiges Drittmaterial unterliegen ebenfalls der genannten Creative Commons Lizenz, sofern sich aus der Abbildungslegende nichts anderes ergibt. Sofern das betreffende Material nicht unter der genannten Creative Commons Lizenz steht und die betreffende Handlung nicht nach gesetzlichen Vorschriften erlaubt ist, ist für die oben aufgeführten Weiterverwendungen des Materials die Einwilligung des jeweiligen Rechteinhabers einzuholen.

Weitere Details zur Lizenz entnehmen Sie bitte der Lizenzinformation auf http://creativecommons.org/licenses/by/4.0/deed.de.

\section{Literatur}

1. Moricke A, Zimmermann M, Reiter A, Henze G, Schrauder A, Gadner H et al (2010) Long-term results of five consecutive trials in childhood acute lymphoblastic leukemia performed by the ALLBFM study group from 1981 to 2000. Leukemia 24:265-284

2. Gupta T, Kannan S, Dantkale V, Laskar S (2011) Cyclophosphamide plus total body irradiation compared with busulfan plus cyclophosphamide as a conditioning regimen prior to hematopoietic stem cell transplantation in patients with leukemia: a systematic review and meta-analysis. Hematol Oncol Stem Cell Ther 4:17-29

3. Peters C, Schrappe M, von Stackelberg A, Schrauder A, Bader P, Ebell W et al (2015) Stem-cell transplantation in children with acute lymphoblastic leukemia: a prospective international multicenter trial comparing sibling donors with matched unrelated donors-the ALL-SCT-BFM-2003 trial. J Clin Oncol 33:1265-1274

4. Boztug H, Sykora KW, Slatter M, Zecca M, Veys P, Lankester A et al (2016) European Society for Blood and Marrow Transplantation analysis of treosulfan conditioning before hematopoietic stem cell transplantation in children and adolescents with hematological malignancies. Pediatr Blood Cancer 63:139-148 